\title{
Staff training improved oral hygiene in patients following stroke
}

\author{
What staff-led oral care interventions are more effective than standard care for \\ individuals after a stroke?
}

\section{Brady M, Furlanetto D, Hunter RV, Lewis S, Milne V. Staff-led interventions for improving oral hygiene in patients following stroke. Cochrane Database Syst Rev 2006; issue 4}

Data sources Reports were identified using the Cochrane Stroke Group and Oral Health Group trials registers, the Cochrane CENTRAL trial register, Cochrane Library, Medline, Cinahl, Research Findings Electronic Register, National Research Register, ISI Science and Technology Proceedings, Dissertation Abstracts and Conference Papers Index, and the reference lists from relevant papers. Authors and researchers in the field were also contacted.

Study selection Randomised controlled trials (RCT) that evaluated one or more interventions designed to improve oral hygiene were selected. Trials based on a mixed population were included, provided it was possible to extract data specific to individuals post-stroke.

Data extraction and synthesis Two review authors independently classified identified trials according to the inclusion and exclusion criteria, assessed the trial quality and extracted data. Clarification was sought from study authors when required.

Results Eight eligible RCT trials were identified but only one provided stroke-specific information. In it, an oral healthcare education training programme delivered to nursing homecare assistants was compared with a delayed training intervention in the control group. Comparisons were made at 1 and 6 months after the intervention, using primary outcome measures of dental plaque and denture plaque, and three secondary outcomes. The data available for the 67 individuals who had suffered a stroke (obtained from the larger cluster-RCT) showed that denture plaque scores were significantly reduced up to 6 months $(P<0.00001)$ after the intervention. Staff knowledge ( $P$ 0.0008) and attitudes (P 0.0001) towards oral care also improved significantly despite high staff turnover rates.

Conclusions Based on one study with a small number of stroke survivors, providing specialist oral care training for carers in a nursing-home setting improves their knowledge of and attitudes towards the provision of oral care. In turn, residents' dentures were cleaner, although other oral hygiene measures did not change. Further evidence relating to oral care interventions is severely lacking, in particular with reference to care in hospital for those following stroke.

\section{Commentary}

This systematic review covers an area where there is very little evidence of good practice. The oral health of older people in general, and of stroke patients in particular, is an area that has been somewhat neglected in the past. It is now receiving more interest in Scotland, particularly as a result of the National Dental Action Plan (formulated in 2005), which identifies older people as a priority group for dental care. The fact that this systematic review was only able to identify one paper that showed data from before and after an oral care intervention for people who had suffered a stroke indicates the lack of research evidence in the field. The study showed that just $1 \mathrm{~h}$ of training for care staff on oral health procedures resulted in a significant improvement in staff knowledge of and attitudes towards oral health, and denture cleanliness. This was maintained even several months after the training, which was encouraging given the high staff turnover in the care sector. The study failed to show any improvement in intra-oral care, however, indicating that this is an area which many care staff still feel is not a priority. Further research will be required to identify the reasons for this.

Many dental staff have been involved in ad hoc training of care staff in the past, and there is a need to develop high-quality, evidence-based packages for this purpose. There is work ongoing in this area, but in the meantime, it is clear from this study that training of care staff can increase their oral health awareness. Any subsequent improvements in oral health will benefit the stroke patient, particularly with regard to speech, nutrition, systemic health and quality of life. On the basis of the study reported here, any input given by dental personnel to care staff might well reap benefits far beyond the oral cavity, and dental staff should be encouraged to work with other healthcare colleagues in a multidisciplinary way to achieve the necessary level of oral health input.

\section{Maura Edwards \\ Public Health Department, National Health Service Ayrshire and Arran, Ayr, Scotland, UK}

Evidence-Based Dentistry (2008) 9, 73. doi:10.1038/sj.ebd.6400593 